\title{
The History of Tuberculosis: Past, Present, and Future
}

\author{
Rohan Prabhu1, Vinodkumar Singh ${ }^{2 *}$ \\ ${ }^{1}$ New York Prebyterian Hospital, Columbia, New York, AL, USA \\ ${ }^{2}$ Department of Anesthesiology and Perioperative Medicine, University of Alabama at Birmingham, Birmingham, AL, USA \\ Email: *vsingh@uabmc.edu
}

How to cite this paper: Prabhu, R. and Singh, V. (2019) The History of Tuberculosis: Past, Present, and Future. Advances in Microbiology, 9, 931-942.

https://doi.org/10.4236/aim.2019.911059

Received: August 13, 2019

Accepted: November 15, 2019

Published: November 18, 2019

Copyright (c) 2019 by author(s) and Scientific Research Publishing Inc. This work is licensed under the Creative Commons Attribution International License (CC BY 4.0).

http://creativecommons.org/licenses/by/4.0/

\begin{abstract}
Tuberculosis (TB), transmitted by the bacteria Mycobacterium tuberculosis has been a global epidemic for years. The evidence for infection with TB is centuries old. It can have an indolent course, and may remain inactive for years in individuals with the possibility of reactivation at any time, causing widespread systemic symptoms. The treatment and management of TB has become more effective over the years. It is estimated that more than one-third of the world population, especially in poor and developing countries are harboring this bacterium with varying manifestations of the disease from no symptoms to life-threatening infections. This manuscript describes the history of the disease and the discoveries. It also includes the recent advances and challenges facing the world and how governments and organizations are working together to control this disease.
\end{abstract}

\section{Keywords}

Tuberculosis, Multi-Drug Resistance, Tuberculosis in India

\section{Introduction}

Tuberculosis (TB) is the third greatest killer worldwide caused by an infectious agent, with over 1.3 million deaths due to TB in 2012 [1]. This is after lower respiratory tract infections and diarrheal diseases. Around 10 million people fell ill due to TB, with an estimated 1 million children [2]. Recent discoveries, healthy living and active treatment modalities along with international efforts has led to a fall in TB incidence by $2 \%$ every year [2]. It is important to have a thorough understanding of the history of the disease, as well as current concerns, such as increasing antibiotic resistance. This will also enable us to develop strategy to fight this disease in the community. We searched the literature relevant to the 
history of TB and the recent advances in the field of treatment. The various official bulletins from WHO were used for demographics and details of worldwide campaigns.

\section{Age of Discovery}

TB is one of the oldest known human diseases and is caused by the bacterium Mycobacterium tuberculosis or Mtb. Evidence of bony tuberculosis dates back to the 8000 B.C. and was also found in spinal cord fragments of Egyptian mummies (circa 2400 B.C.) [3]. Around 460 B.C., Hippocrates (Figure 1) recognized TB as a frequently fatal disease, warning doctors against treating citizens suffering from late stage $\mathrm{TB}$, as their inevitable deaths could ruin their reputation [4].

Over the centuries, TB has had many names, including psithisis pulmonaris, white plague, and consumption. The name consumption came from the fact that the infection seems to "consume" the patient. Dutch scientist, Sylvius de la Boe, provided the first pathological and anatomical descriptions of the disease in 1679 by identifying tubercles as a consistent and characteristic pathologic manifestation in the lungs of patients with TB [5]. His reports were later published in $O p$ era Medica, describing how the disease usually affected the lungs, but later progressed to cavitary lesions and abscesses [5]. He was the first to cite an association between phthisis and a disease of the lymph nodes of the neck, termed scrofula (Figure 2). Scrofula was also called the "king's evil" as it was widely believed (since the days of Clovis of France, 487 AD) that the kings of England and France could cure scrofula simply by touching those affected [6]. Spinal tuberculosis, dubbed "Pott's disease", was first described in 1779 by the British surgeon Percivall Pott [7]. TB can affect any organ, although lungs are most commonly affected and patients frequently present with symptoms such as cough, chest pain, fatigue, and unintentional weight loss.

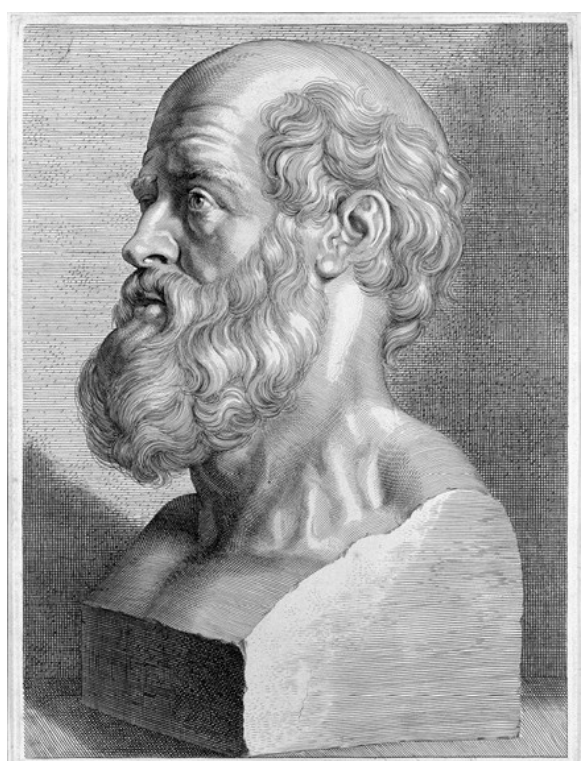

Figure 1. Hippocrates. 


\section{Advances in Diagnosis}

Benjamin Marten, who described tuberculosis as a disease caused by "wonderfully minute living creatures", first recognized TB as a communicable disease [8]. He also reported that close contact with a person with TB, over an extended period, was required to contract the disease [9]. In 1865, a French military physician, Jean-Antoine Villemin showed that TB could be transmitted from humans to cattle and from cattle to rabbits [10]. He also questioned the commonly held belief that the disease arose spontaneously and instead believed that the disease came from a specific microorganism [11]. In 1882, Robert Koch developed a special staining technique to visualize $M t b$ and described the transmission of tuberculosis through droplets released with sneezing or coughing [12]. In 1901, Koch addressed the British Congress (Figure 3) stating, "There is no disease which inflicts such deep wounds on mankind as this" [13]. Koch was one of the

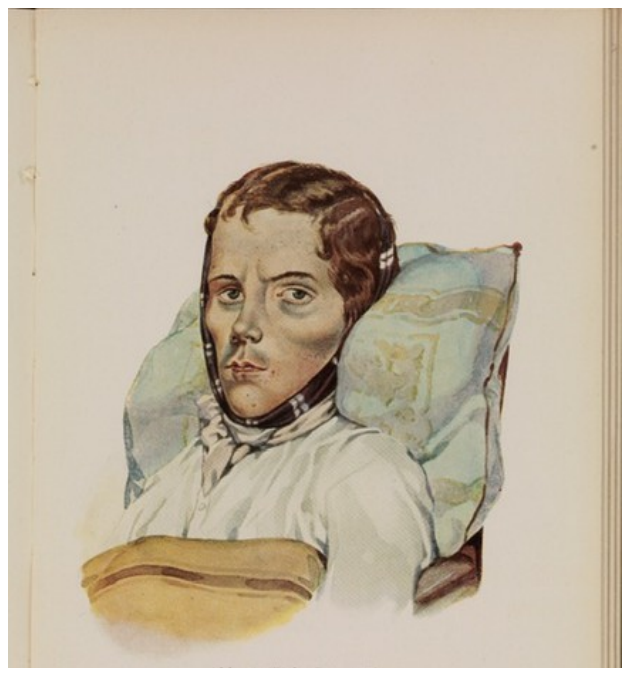

Figure 2. Scrofula.

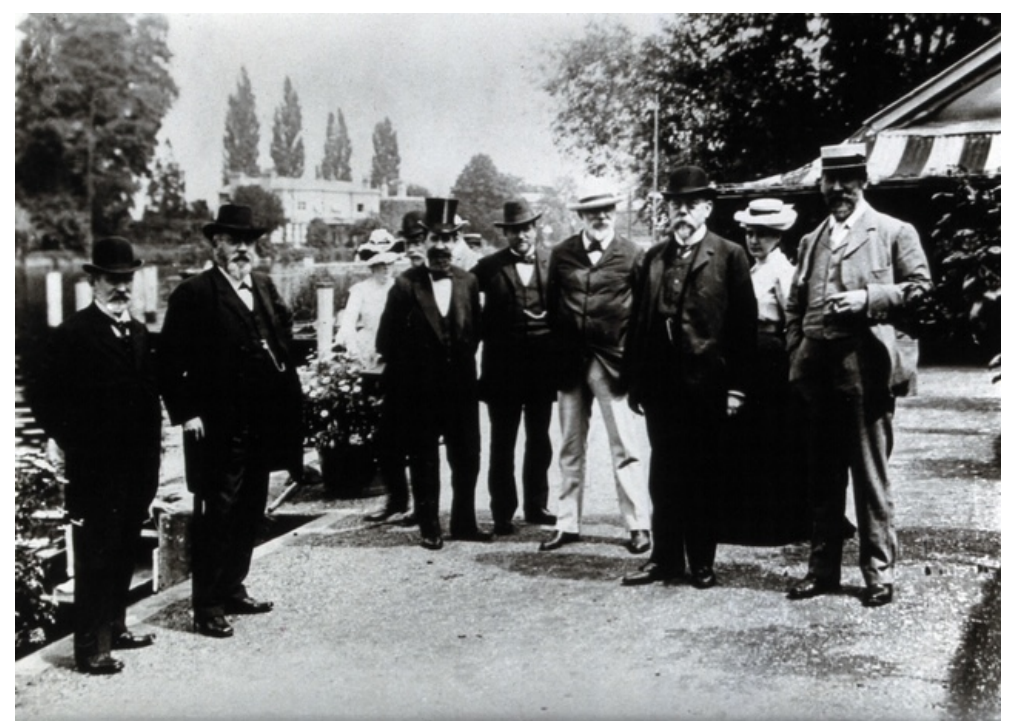

Figure 3. Koch_1901. 
most influential figures in the fight against TB, who later received the Nobel Prize in Physiology or Medicine in 1905 for his discoveries [14].

The discovery of Lowenstein-Jensen (LJ) agar marks another important milestone in the diagnosis of TB. This agar had various ingredients to optimize growth of TB like Malachite green, glycerol, asparagine, potato starch, coagulated eggs, and mineral salt solution [15]. Penicillin and Nalidixic acid were often added to prevent growth of bacteriae on the agar [15]. When grown on the agar, $M t b$ appears as a brown, granular colony. Unfortunately, due to the slow growth rate of $M t b$, the incubation period may last an average of four weeks.

Around the same time as the discovery of TB growth media, the Housing of the Working Class Act was enacted in the United Kingdom in 1885, which led to an improvement in living standards and a decrease in overcrowding in certain areas [16]. The benefits from this were recognized and similar laws and rules were passed in many other countries.

The idea of a sanatorium for people who were diagnosed with tuberculosis stemmed from physicians like Hermann Brehmer who would instruct patients to go to "healthier climates" to improve their health [17]. Sanatoriums were developed based on the belief that good rest, nutrition, and fresh air would allow patients to fight off a TB infection [18]. The famous sanatorium, Brehmersche Heilanstalt fur Lungenkranke in Gorbersdorf (Sokolowsko), Silesia (now Poland) was thus opened in 1856. Over the next century, thousands of sanatoria were founded throughout Europe (Figure 4) and the United States. In 1884, Edward Livingston Trudeau opened the first sanatorium in the United States [19].

\section{Discovery of Drugs and Tuberculosis Treatment}

The late 1800s and early 1900s mark a transition point in the history of TB, where mortality from the disease steadily decreased. This happened even before the introduction of antibiotics and immunization against TB. In 1895, X-rays or Roentgenogram (Figure 5) was discovered by German physicist Wilhelm Konrad von

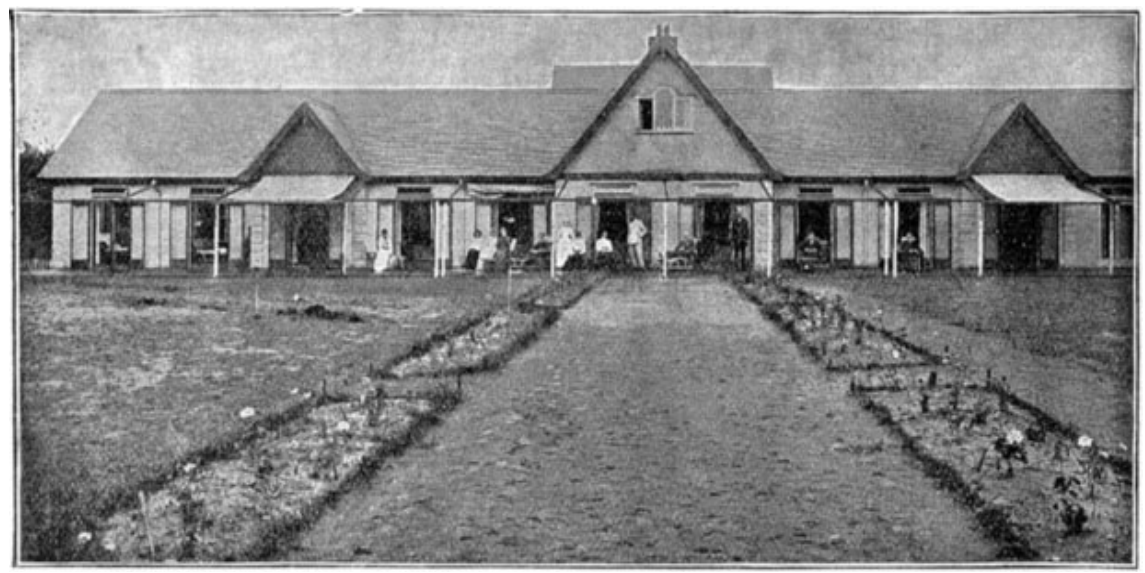

Figure 4. Crooksbury sanatorium (1). 
Roentgen. With the help of Chest X-rays, it became easier to diagnose pulmonary tuberculosis as well as follow-up these patients and those suspected to have TB [5]. Roentgen received the Nobel Prize in Physics in 1901 for this discovery.

In 1906, French physician, Leon Charles Albert Calmette (Figure 6), and immunologist, Camille Guerin, developed the Bacillus Calmette-Guerin (BCG) vaccine against TB [20]. Immunizations were first carried out in Paris in 1921, especially in children [21]. Mass immunization was carried out starting from military recruits and institutionalized people; and then spreading to the whole population at risk of developing TB. Later data suggested that while this vaccine

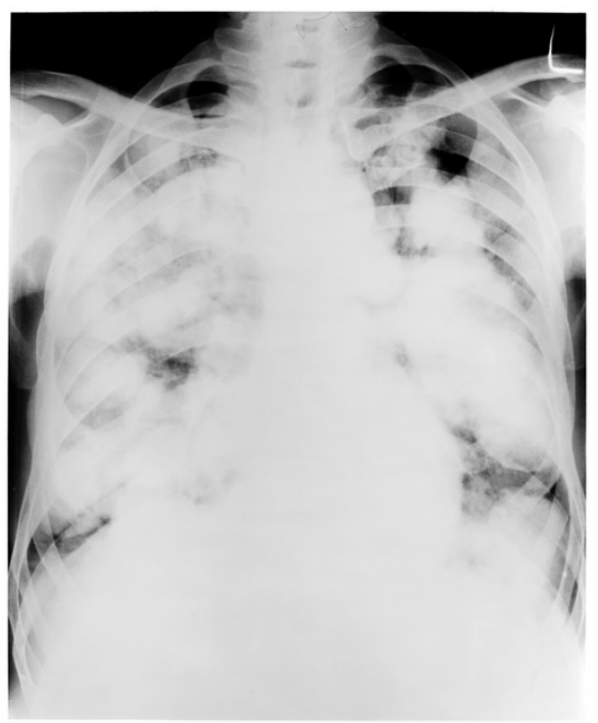

Figure 5. Chest_Xray_TB.

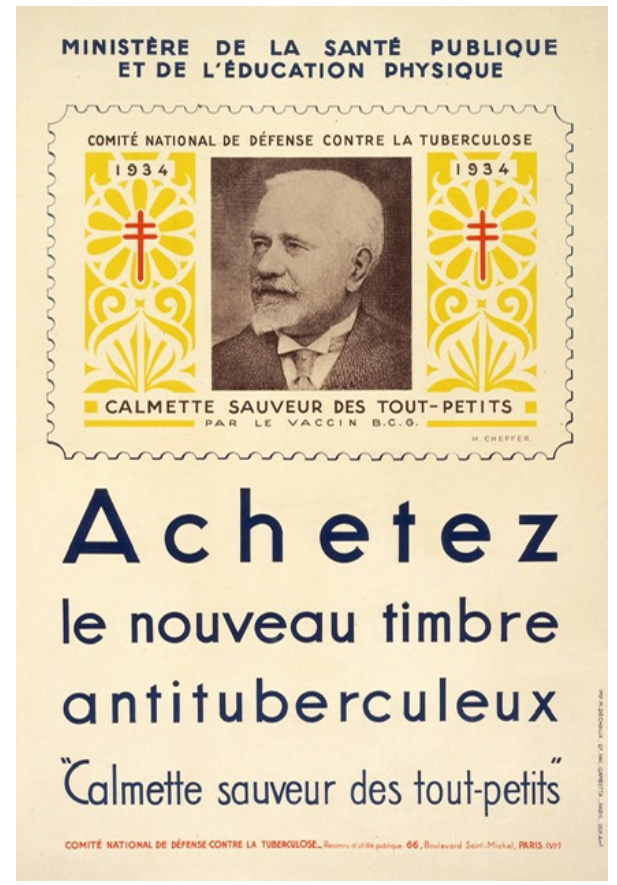

Figure 6. Leon Charles Albert Calmette. 
may be helpful in preventing serious forms of childhood TB, it does not have the same effect in adults [5]. Thus, the primary treatment remains prevention of transmission and treatment of active cases with anti-tubercular drugs. In Great Britain, the Medical Research Council was established in 1913 with its major focus to tackle TB [22]. Prior to the discovery of anti-TB drugs, surgical techniques like plombage and pneumonectomy were the only medical options available to patients. "Plombage" is a surgical method to treat cavitary tuberculosis of the upper lobe of lung and refers to the insertion of an inert substance in the pleural space to compress the cavitary lesion [23].

In 1943, the antibiotic streptomycin was developed by persistent work by Selman Waksman. He was honored for his discovery with a Nobel Prize in Physiology or Medicine in 1952 [24]. This was the first antibiotic shown to be effective against TB. The inability to use it through the oral route and its toxicity profile made it inconvenient to use for the longer treatment duration. It was also realized that TB could not be treated with one antibiotic alone due to the development of resistance due to mutation of ribosomal target sites.

In 1949, p-aminosalicylic acid was discovered followed later in 1952 with Isonicotinic acid hydrazide or Isoniazid. Isoniazid's bactericidal properties made it a favored first line agent against TB, but resistance was documented. Then, in the 1950s, other effective antibiotics like pyrazinamide, ethambutol, cycloserine and ethambutol were developed [5]. Combination therapy using these antibiotics showed promise with increased cure rates and decreased mortality. Over the next few years, more drugs were developed, such as ethambutol and rifampicin.

Major problems that remain in TB endemic countries included patient noncompliance and the development of multi-drug resistance. Currently, the most popular combination regimen consists of using four drugs: Rifampin, Isoniazid, Pyrazinamide, and Ethambutol (RIPE therapy) for 2 months, followed by Rifampicin and Isoniazid for another 4 months. This is generally adequate to treat uncomplicated pulmonary $\mathrm{TB}$, but longer treatments and different combinations may be needed on a case-by-case basis.

\section{Worldwide Campaigns}

In 1993, the World Health Organization (WHO) classified TB as a worldwide epidemic with approximately one-third of the world's population infected [25]. While 13 of the 15 countries with the highest TB incidence rates are in Africa, more than half of new cases now come from five Asian countries that include Bangladesh, India, Indonesia, Pakistan, and the Philippines [26). This has shifted public health efforts to the Southeast Asian Region (SEAR), where communicable diseases result in $42 \%$ of Disability-Adjusted Life Years (DALY) lost [27]. DALY is a global measure to compare overall disease burden expressed as the number of years lost due to health reasons, disability or early death.

Over the past decade, many countries have begun implementing the Directly Observed Treatment Short-Course (DOTS) program, which is currently the 
most cost-effective way to stop the spread of tuberculosis [28]. DOTS is a sixmonth regimen where patients are put under close observation to make sure that proper medication is being taken and is designed to decrease the prevalence of multi-resistant strains of $M t b$. There are five key components to the DOTS program [28]:

1) Political commitment with increased and sustained financing;

2) Case detection through quality-assured bacteriology;

3) Standardized treatment, with supervision and patient support;

4) An effective drug supply and management system;

5) Monitoring and evaluation system, and impact measurement.

Between 1995 and 2008, more than 36 million TB patients were successfully treated using the DOTS program, with approximately 6 million deaths averted [26].

Other worldwide campaigns include those led by The United Nations Children's Emergency Fund (UNICEF), which has initiatives focused on improving child survival growth and development, water sanitation and hygiene, and improved access to vaccines [29]. Additionally, organizations such as "Americares" are working to provide medical aid and humanitarian relief in cases of environmental emergencies, such as monsoons [30]. While there are a large number of government programs and non-government organizations (NGO) that help to provide healthcare and relief, there is still a lot of work that can be done to further improve health care access and provide support for underprivileged and vulnerable populations.

\section{Multi-Drug Resistant Tuberculosis}

Multi-Drug Resistant TB (MDR-TB) is the development of resistance to the two most powerful anti-TB drugs Isoniazid and Rifampin [31]. It was recognized early in the campaign of treatment of TB that inappropriate or incorrect use of antimicrobial drugs, or the use of ineffective formulations (single agent, poor quality of drugs or bad storage), could cause development of resistance, which then can be transmitted [31]. The incidence of MDR-TB has slowly increased recently gaining worldwide attention. In some cases, even more severe drug resistance can develop with additional resistance to more anti-TB drugs leading to Extensively Drug-Resistance TB (XDR-TB) [19]. WHO reports that of the 480,000 people worldwide infected with MDR-TB, approximately $10 \%$ have XDR-TB [31]. Totally drug-resistant tuberculosis (TDR-TB) is now emerging in many countries and is resistant to all the major antibiotics used in the treatment of TB [32].

For antibiotic susceptible TB, diagnosis has become easier with molecular or culture-based studies. To effectively control TB and avoid MDR-TB or TDR-TB, the following recommendations should be followed [33]:

- Curing the patient on the first detection;

- Providing access to diagnosis and drugs; 
- Adequate infection control in treatment facilities; and

- Appropriate use of second line drugs for TB.

This will reduce the number of treatment failures and the generation of drug resistance in the organisms. Most patients should be treated in the community to avoid concentration in the treatment facilities and development of resistance and second line drugs should be used cautiously.

\section{Tuberculosis in India}

India has the largest number of active TB cases in the world, with an estimated $40 \%$ of the Indian population being a TB carrier [34]. While the incidence and mortality rate of TB in India has decreased over the years, there has been an increasing incidence of MDR-TB in India [35]. Among new and previously treated patients, the prevalence of MDR-TB was $2.2 \%$ and $15 \%$, respectively [35]. Treatment of all latent TB patients in India is neither effective nor feasible, which has led to treatment focused on latent cases only if there are other risk factors involved, such as immunocompromised or co-infection. Immunocompromised individuals, such as patients with HIV, are much more susceptible to developing active TB infection due to their weakened immune systems. TB-HIV co-infections have risen over the last decade and have led to public health campaigns focused on increasing both awareness and testing (Figure 7) [26]. Other vulnerable populations include children less than two years old who are household contacts of patients with smear-positive pulmonary TB and patients who need glucocorticoid therapy or immunosuppressive therapy [36].

Reasons for the high prevalence of TB in India are manifold: poor access to health care, absence of uniform health insurance, poverty, poor infection control measures, poor hygiene, and overcrowding along with lack of adequate ventilation and sunlight [37]. Health care workers in India are also prone to higher TB infection rates, due to a lack of adequate Personal Protective Equipment (PPE)

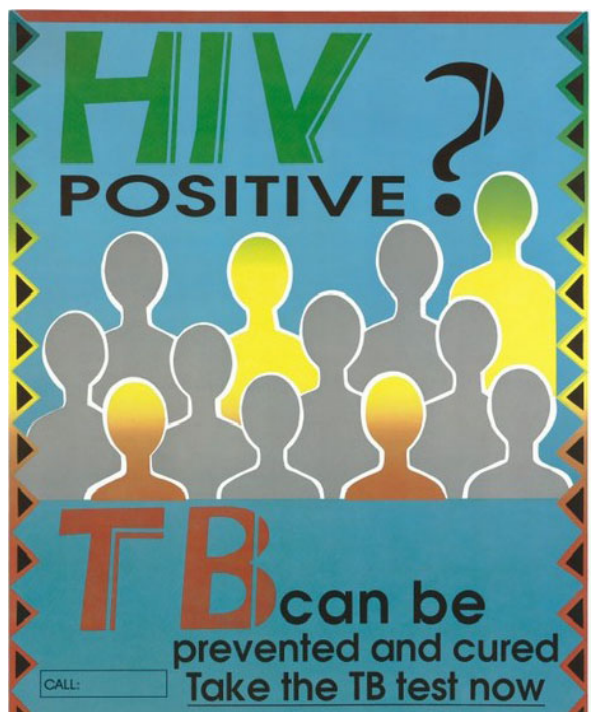

Figure 7. HIV_TB_Test. 
such as gloves and masks [37]. Lack of patient knowledge about the spread of TB has led to a lack of good cough etiquette and inadequate hygiene [37].

Compliance with treatment has been a major issue in India due to the lack of knowledge about the disease in poor populations. Treatment is often started, but the antibiotic course is not finished leading to increased relapse of the disease and rise in the number of TB resistant microorganisms. Control of the disease is expected to become increasingly difficult as the life expectancy in India increases, because elderly individuals are more susceptible to TB infection [38].

Worldwide TB campaigns have been targeting improved drug compliance with moderate success. The DOTS program and the Revised National Tuberculosis Control Program (RNTCP) have laid out specific initiatives to control the spread of TB in India [34]. This includes political and administrative commitment, systematic monitoring, supervised treatment, and quality diagnosis [34]. Since the implementation of some of these initiatives, TB prevention, diagnosis, and treatment interventions have saved an estimated 37 million lives and the TB mortality rate fell by $45 \%$ and the prevalence rate by $41 \%$ between 1990 and 2013 [39]. While India was on track to meet the Millennium Development Goals, there is a long way to go in order to reduce the prevalence and transmission of the disease [39].

\section{Future Problems and Advances}

Increasing drug resistance and drug compliance remain two major future problems in the worldwide prevention and control of TB. Due to the slow growth rate of TB, results from sputum smear microscopy often take several weeks during which the patient can transmit the infection to many other community members. There have been newly approved automated tests such as Xpert, that are able to rapidly assess resistance to antibiotics such as rifampicin, but these tests have not yet become mainstream [40]. In the modern era, the purified protein derivative (PPD) skin test has traditionally been used to help diagnose TB infection by injecting TB antigens into the patient's skin [41]. A positive PPD test would manifest itself as a red induration at the site of injection (Figure 8).

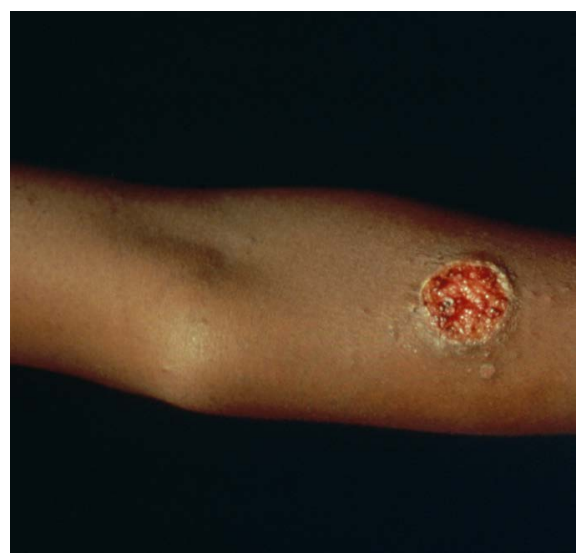

Figure 8. Positive PPD test. 
Instead of the TB skin test, interferon gamma release assay (IGRA), a blood test can also be done to diagnose TB infection [41].

As individuals who are HIV positive are much more likely to develop the active form of TB, it is also important for health officials to focus efforts on HIV prevention by raising awareness about the importance of contraception, seeking out treatment for Sexually Transmitted Diseases (STDs), and providing peer counseling for high-risk individuals. Finally, it will be important to continue raising awareness and to provide funding for successful programs to decrease the prevalence of TB around the world.

The WHO End Tuberculosis strategy was adopted in 2014 with targets to reduce TB deaths by $90 \%$ and to cut new cases by $80 \%$ between 2015 and 2030. It also wants to ensure that families are not burdened by the cost of treatment of TB. It has also set a 2035 target of $95 \%$ reduction in deaths and $90 \%$ decline in $\mathrm{TB}$ incidence that is similar to the levels in low TB incidence countries today [42].

\section{Conclusion}

A combined effort of various governments, international organizations along with recent discoveries in diagnosis and treatment will lead to achievement of the above goals and control in the disease that has a huge economic implication on already compromised societies and countries. We have seen huge medical successes in the recent years like Small pox, polio and measles; and we hope to continue our fight against diseases like TB.

\section{Acknowledgements}

The authors would like to acknowledge the work of Emma O'Hagan, MLIS, Assistant Professor and Clinical Research and Education Librarian, Department of Anesthesiology and Perioperative Medicine, University of Alabama at Birmingham, Birmingham, AL. The authors thank Brittany Nelson Hermecz, MD, a diagnostic radiology resident at the University of Alabama at Birmingham for her initial background research on this project.

Figures/Images courtesy: Wellcome Library, London.

\section{Conflicts of Interest}

The authors declare no conflicts of interest regarding the publication of this paper.

\section{References}

[1] Sulis, G., Roggi, A., Matteelli, A. and Raviglione, M. (2014) Tuberculosis: Epidemiology and Control. Mediterranean Journal of Hematology and Infectious Diseases, 6, e2014070. https://doi.org/10.4084/mjhid.2014.070

[2] World Health Organization (2018) Tuberculosis Key Facts 2018. https://www.who.int/en/news-room/fact-sheets/detail/tuberculosis 
[3] Zink, A., Sola, C., Reischl, U., Grabner, W., Rastogi, N., Wolf, H. and Nerlich, G. (2003) Characterization of Mycobacterium tuberculosis Complex DNAs from Egyptian Mummies by Spoligotyping. Journal of Clinical Microbiology, 41, 359-367. https://doi.org/10.1128/JCM.41.1.359-367.2003

[4] Daniel, T.M. and Iverson, P.A. (2015) Hippocrates and Tuberculosis. The International Journal of Tuberculosis and Lung Disease, 19, 373-374.

https://doi.org/10.5588/ijtld.14.0736

[5] Herzog, H. (1998) History of Tuberculosis. Respiration, 65, 5-15. https://doi.org/10.1159/000029220

[6] Moulis, G. (2012) Scrofula, the King's Evil. Canadian Medical Association Journal, 184, 1061. https://doi.org/10.1503/cmaj.111519

[7] Sternbach, G. (1996) Percivall Pott: Tuberculosis Spondylitis. Journal of Emergency Medicine, 14, 79-83. https://doi.org/10.1016/0736-4679(95)02053-5

[8] Doetsch, R. (1978) Benjamin Marten and His "New Theory of Consumptions." Micriobiolical Reviews, 42, 521-528.

[9] Keers, R.Y. (1978) Pulmonary Tuberculosis: A Journey Down the Centuries. Bailliere-Tindall, London.

[10] Ferlinz, R. (1995) Die Tuberkulose in Deutschland und das deutsche Zentralkomitee zur Bekämpfung der Tuberkulose. Pneumologie, 49, 617-632.

[11] Löffler, W. (1958) Geschichte der Tuberkulose. In: Hein, J., Kleinschmidt, H. and Uehlinger, E., Eds., Handbuch der Tuberkulose, Stuttgart, Thieme.

[12] Ayvazian, L.F. (1993) History of Tuberculosis. In: Reichman, L.B. and Hershfield, Eds., Tuberculosis, Dekker, New York.

[13] Koch, R. (1901) The Fight against Tuberculosis in Light of the Experience Gained in the Successful Combat of Other Infectious Diseases. Boston Medical and Surgical Journal, 145, 109-115. https://doi.org/10.1056/NEJM190108011450501

[14] Sakula, A. (1979) Robert Koch (1843-1910): Founder of the Science of Bacteriology and Discoverer of the Tubercle Bacillus. British Journal of Diseases of the Chest, 73, 389-394. https://doi.org/10.1016/0007-0971(79)90078-0

[15] Hardy Diagnostics. Lowenstein Jensen (LJ) Agar Instructions for Use. https://catalog.hardydiagnostics.com/cp_prod/Content/hugo/LJMedia.htm

[16] UK Government. Housing of the Working Classes Act 1885. http://www.legislation.gov.uk/ukpga/Vict/48-49/72/enacted

[17] Ferlinz, R. (1995) Die Tuberkulose in Deutschland und das deutsche Zentralkomitee zur Bekämpfung der Tuberkulose. Pneumologie, 49, 617-632.

[18] Pratt, J.H. (1944) The Evolution of Rest Treatment of Pulmonary Tuberculosis. The American Review of Tuberculosis, 50, 185-201.

[19] Sigerist, H.E. (1961) A History of Medicine. Oxford University Press, London.

[20] Bochalli, R. (1959) Die Entwicklung der Tuberkuloseforschung in der Zeit von 1878 bis 1959. Stuttgart, Thieme.

[21] Luchino, F. (1982) BCG Vaccination. The American Review of Respiratory Disease, $125,70-72$.

[22] Bryder, L. (2014) The Medical Research Council and Treatments for Tuberculosis before Streptomycin. Journal of the Royal Society of Medicine, 107, 409-415. https://doi.org/10.1177/0141076814548663

[23] Sakula, A. (1983) Carlo Forlanini, Inventor of Artificial Pneumothorax for Treatment. Thorax, 38, 326-332. https://doi.org/10.1136/thx.38.5.326 
[24] Sakula, A. (1988) Selman Waksman (1888-1973), Discoverer of Streptomycin: A Centenary Review. British Journal of Diseases of the Chest, 82, 23-31. https://doi.org/10.1016/0007-0971(88)90005-8

[25] Davies, P.D. (2000) Tuberculosis: The Global Epidemic. Journal of the Indian Medical Association, 98, 100-102.

[26] Sandhu, G. (2011) Tuberculosis: Current Situation, Challenges, and Overview of Its Control Programs in India. Journal of Global Infectious Diseases, 3, 143-150. https://doi.org/10.4103/0974-777X.81691

[27] Gupta, I. and Guin, P. (2010) Communicable Disease in the South-East Asia Region of the World Health Organization: Towards a More Effective Response. Bulletin of the World Health Organization, 88, 199-205. https://doi.org/10.2471/BLT.09.065540

[28] Sandiford, P. (1999) WHOs DOTS Strategy. Directly Observed Therapy. The Lancet, 353, 755. https://doi.org/10.1016/S0140-6736(05)76125-0

[29] UNICEF India (2016) What We Do. http://unicef.in/WhatWeDo

[30] Americares (2016) What We Do. http://www.americares.org/what-we-do

[31] Centers for Disease Control and Prevention (CDC) Tuberculosis. https://www.cdc.gov/tb/publications/factsheets/drtb/mdrtb.htm

[32] Klopper, M., Warren, R.M., Hayes, C., Gey van Pittius, N.C., Streicher, E.M., Müller, B., Sirgel, F.A., Chabula-Nxiweni, M., Hoosain, E., Coetzee, G., van Helden, P.D., Victor, T.C. and Trollip, A.P. (2013) Emergence and Spread of Extensively and Totally Drug-Resistant Tuberculosis, South Africa. Emerging Infectious Diseases, 19, 449-455. https://doi.org/10.3201//EID1903.120246

[33] Lobue, P. (2006) Extensively Drug-Resistant Tuberculosis. Current Opinion in Infectious Diseases, 12, 1311-1318.

[34] Pai, M., Kalantri, S., Aggarwal, A.N., Menzies, D. and Blumberg, H. (2006) Nosocomial Tuberculosis in India. Emerging Infectious Diseases, 12, 1311-1319. https://doi.org/10.3201/eid1209.051663

[35] Prasad, R., Gupta, N. and Singh, M. (2014) Multidrug Resistant Tuberculosis: Trends and Control. Indian Journal of Chest Diseases and Allied Sciences, 56, 237-246.

[36] Agarwal, R. (2005) Treatment of Latent Tuberculosis Infection in India: Is It Worth the Salt? Lung India, 22, 105-106.

[37] Aggarwal, A. (2009) Tuberculosis Transmission at Healthcare Facilities in India. Lung India, 26, 33-34. https://doi.org/10.4103/0970-2113.48893

[38] Bying-Maddick, R. and Noursadeghi, M. (2016) Does Tuberculosis Threaten Our Ageing Populations? BMC Infectious Diseases, 16, 119.

https://doi.org/10.1186/s12879-016-1451-0

[39] World Health Organization (2014) Global Tuberculosis Report 2014. http://www.who.int/tb/publications/global_report/gtbr14_executive_summary.pdf

[40] McNerney, R. and Zumla, A. (2015) Impact of the Xpert MTB/RIF Diagnostic Test for Tuberculosis in Countries with a High Burden of Disease. Current Opinion in Pulmonary Medicine, 21, 304-308. https://doi.org/10.1097/MCP.0000000000000161

[41] Al-Orainey, I. (2009) Diagnosis of Latent Tuberculosis: Can We Do Better? Annals of Thoracic Medicine, 4, 5-9. https://doi.org/10.4103/1817-1737.44778

[42] WHO (2019) WHO End TB Strategy. https://www.who.int/tb/post2015_strategy/en/ 